\title{
Effects of host size and laboratory rearing on offspring development and sex ratio in the solitary parasitoid Agrothereutes lanceolatus (Hymenoptera: Ichneumonidae)
}

\author{
TAKATOSHI UENO \\ Institute of Biological Control, Faculty of Agriculture, Kyushu University, Fukuoka 812-8581, Japan; \\ e-mail: ueno@grt.kyushu-u.ac.jp
}

Key words. Hymenoptera, Ichneumonidae, Cryptinae, Agrothereutes lanceolatus, host quality, host selection, laboratory rearing, idiobiont, sex allocation

\begin{abstract}
Agrothereutes lanceolatus is a solitary ectoparasitoid attacking mature larvae, prepupae and pupae of tortricid and pyralid moths, including Homona magnanima, which is an important pest of tea. Adult A. lanceolatus are sexually dimorphic and vary considerably in size in the wild, suggesting they parasitize a variety of hosts of different sizes. Here, the effects of host size on offspring survival, size and sex ratio in A. lanceolatus were investigated. Wild-caught and laboratory-reared parasitoids were used to identify the effects of laboratory rearing on the above three biological parameters. The percentage of hosts producing parasitoid offspring was not dependent on host size regardless of parasitoid generation. Offspring survival was lower in laboratory-reared generations than the F1 generation. Large parasitoids emerged from large hosts. The offspring sex ratio (proportion of females emerging) was positively correlated with host size. The sex ratio at the egg stage (= primary sex ratio) inferred from maternal fertilization act also changed in response to host size, although there was imperfect control of female egg deposition. Thus, the observed sex-ratio resulted from maternal control of offspring sex ratio. A striking difference was detected in the offspring sex ratio in the different generations. Offspring sex ratio was more male-biased in laboratory reared generations. These results suggest that inbreeding depression caused a reduction in offspring survival and the male-biased sex ratio in the laboratory. The sex-ratio difference could result either from the difference in host size in the field and laboratory or from the production of diploid males in the laboratory.
\end{abstract}

\section{INTRODUCTION}

Female parasitoids in the field will encounter many potential hosts. However, hosts are not always suitable for oviposition; some may be nutritionally inferior, being unsuitable for offspring development. Therefore, female parasitoids upon encountering a potential host assess its quality before deciding to lay eggs (Vinson \& Iwantsch, 1980a, b; Godfray, 1994; Ueno, 2000; Hajek, 2004; Rehman \& Powell, 2010).

Host-size-dependent host-selection is common among parasitoids because the size of hosts is a determinant of host quality (Charnov \& Skinner, 1984; Godfray, 1994; Goubault et al., 2004; Rehman \& Powell, 2010; Liu et al., 2011). For idiobiont parasitoids in which hosts are permanently paralyzed upon parasitization (= oviposition), the amount of the host resources available for the parasitoid offspring is determined by the size of the hosts at oviposition. Thus, host size reflects the size of the resulting offspring, and offspring size largely affects the reproductive success of male and female parasitoids (King, 1987; Ueno, 1999a; Heimpel \& Lundgren, 2000; Henter, 2004; Wang \& Messing, 2004; Traynor \& Mayhew, 2005; Liu et al., 2011).

Host size is also an important factor influencing parasitoid sex ratio. For haplo-diploid hymenopteran parasitoids, the females can selectively lay female or male eggs by controlling the access of sperm to eggs; during oviposition, they are able to manipulate the release of sperm from the spermatheca as the eggs pass through her genital tract (Godray, 1994; Quicke, 1997). Unfertilized and fertilized eggs develop into males and females, respectively. Many parasitoid wasps respond to host size by changing sex allocation and lay unfertilized male eggs in small hosts because small females are more heavily penalized than small males (King, 1987, 1993; Ueno, 1998a, 1999a; Ode \& Heinz, 2002). Thus, host size affects parasitoid host selection, development, reproductive success and offspring sex ratio. How host size influences these biological parameters is intimately associated with parasitoid life history and biology.

Also, understanding such host-size effects is important for biological control using parasitoids (Jervis \& Kidd, 1996; Heimpel \& Lundgren, 2000; Ode \& Heinz, 2002; Hajek, 2004). Laboratory hosts are commonly used for mass propagation of parasitoids for biological control. Such hosts are used because natural hosts are often difficult to rear in the laboratory or are costly to mass-rear. Thus it is advantageous to use laboratory hosts because they are easy and cheap to mass-rear. The suitability of such laboratory hosts for parasitoid production should be investigated prior to their selection. Host size is again important in assessing the suitability of a laboratory host as it may differ from that of natural hosts.

Here the basic biology of an ectoparasitoid wasp, Agrothereutes lanceolatus (Hymenoptera: Ichneumonidae), was studied by focusing on the effects of host size on $A$. 
lanceolatus. This parasitoid is an important natural enemy of several important lepidopteran pests in tea and fruit orchards (Townes et al., 1965; Yasumatsu \& Watanabe, 1965; Mao \& Kunimi, 1991). It is a member of the subfamily Cryptinae and is widely distributed in Japan. Host records include at least 13 species belonging to two families of Lepidoptera. Field studies indicate that A. lanceolatus is multivoltine and the adult parasitoid is active from early spring to late autumn.

The life history of $A$. lanceolatus is poorly known. Preliminary observations have shown that female $A$. lanceolatus have a well-developed venom gland. The venom glands of parasitoid wasps are the source of a secretion that paralyses the host. In fact, $A$. lanceolatus paralyzes hosts before oviposition, which from then on cease growing (Ueno, 2000). Thus, it is possible that $A$. lanceolatus gauges host size at oviposition. Like many other ectoparasitoid wasps, A. lanceolatus is a typical idiobiont (Ueno, 2002). Also, it is a host-feeding parasitoid and produces anhydropic eggs (Ueno, 1999b).

A. lanceolatus is a sexually-dimorphic species in which the adult males are more slender and have longer legs than adult females. Wild $A$. lanceolatus vary in adult size. Based on this observation and host records, the size of the hosts of wild A. lanceolatus vary, which should result in hostsize related host selection by this parasitoid, if host size affects the size of parasitoid offspring. In a previous paper, I have demonstrated that, in A. lanceolatus, the size of adult males does not affect male longevity whereas adult female longevity is positively associated with their size (Ueno, 1999b). Sexually asymmetric effects of adult parasitoid size on longevity indicate that female $A$. lanceolatus may control the sex-ratio of their offspring in response to host size as the host size model predicts (Charnov et al., 1981; King, 1993).

Accordingly in the present study I investigate the relationships between host size and offspring performance (i.e. development time, survival, size) in A. lanceolatus when a laboratory host, Galleria mellonella, is used. I also determine the sex ratio of the offspring that emerged from hosts of different sizes. In addition, the effects of rearing on a laboratory host on parasitoid performance and sex ratio were assessed. Based on the results, the relationships between host size and biology of A. lanceolatus are discussed.

\section{MATERIAL AND METHODS}

\section{Parasitoid and host}

The method of rearing A. lanceolatus is described by Ueno (1999b, 2002). Newly emerged females of A. lanceolatus were individually placed in plastic containers $(10 \mathrm{~cm}$ in diameter, 4.5 $\mathrm{cm}$ in height) with a male. Tissue paper saturated with diluted honey was placed in the containers as food and replaced twice a week. Male parasitoids were removed after 2-4 days. This treatment was enough to ensure that all female parasitoids were inseminated. The containers were kept at $20 \pm 1{ }^{\circ} \mathrm{C}$ under a photoperiod of $16 \mathrm{~L}: 8 \mathrm{D}$.

The laboratory host G. mellonella was reared on an artificial medium. This host was chosen because it is highly suitable for rearing a number of idiobiont ichneumonid parasitoids (Ryan, 1971; Sandlan, 1982; Ueno, 1999a). In fact, a previous study indicates that $G$. mellonella is suitable because female A. lanceolatus readily selects it for oviposition and because $80 \%$ of its offspring survive in this host (Ueno, 2002).

Newly formed cocoons of G. mellonella were selected and collected from rearing cages, and kept at $5 \pm 1{ }^{\circ} \mathrm{C}$ until used in the experiments. Cocoons containing prepupa were used in the tests. Various sizes of $G$. mellonella cocoon were obtained by changing larval density.

\section{Host size}

In order to determine the host-size/host-suitability relationship, hosts of various weights were used. Two groups of females were used in this experiment; wild-caught and laboratory reared females. Wild-caught females were collected in Nagoya City, Japan, using an insect net and were used in experiments 1-2 days later. Wild-caught females readily accepted and oviposited in $G$. mellonella. Newly emerged females in the F3-F4 generations of the parasitoid culture were the 'laboratory-reared' parasitoids. Laboratory-reared females used in the experiments were 7-10 days old. Before testing recently emerged females they were allowed to mate once and then given access to 2 healthy host cocoons for $2 \mathrm{~h}$ every 2 days. Host cocoons used in this pre-experimental treatment were randomly selected from rearing cages, which resulted in cocoons of different sizes being presented to each female parasitoid.

Tests were conducted by presenting each female with 3-4 unparasitized healthy hosts, which had previously been weighed using an electronic balance (Shimazu AUY220, Japan). In the present study, host weight is considered to be synonymous with host size. Behavioural responses of females to hosts were directly observed. Observations started when a female mounted a host and terminated when she left the host. Each host attacked was removed immediately after the female left the host, thus preventing multiple parasitism. Preliminary observations revealed that hosts that had been attacked once contained one parasitoid egg.

Hosts attacked were kept at $20 \pm 0.5^{\circ} \mathrm{C}$ under a photoperiod of $16 \mathrm{~L}: 8 \mathrm{D}$. The sex of emerging parasitoids was recorded. Forewing length of parasitoids was measured and used as an index of offspring size. The wing length was measured under a stereoscopic microscope. Hosts were kept for a maximum of 40 days. All females produced at least one female offspring. In all, 14 wild-caught and 18 laboratory-reared females were used in this experiment.

\section{Primary sex-ratio}

This experiment was performed to determine whether the offspring sex-ratio/host size relationship recorded in the above experiment was due to maternal control of the offspring sex-ratio via fertilization control. To do this, abdominal tip movements of ovipositing $A$. lanceolatus were observed. A number of previous studies have shown that movements of the tip of the abdomen of ovipositing females are of two types; interrupted and continuous, and can be used to determine the primary sex ratio, i.e., sex ratio at the egg stage (Strand, 1989; Ueno, 1995). The former movement is involved in fertilization and hence production of female eggs and the latter of male eggs.

In this experiment, 14 wild caught females were used. They were provided with host cocoons and their response to cocoons recorded. Each female was observed ovipositing 3 times. When a female attacked a host cocoon, abdominal and ovipositor movements during oviposition were carefully observed under a binocular microscope. After the observations, parasitized hosts were reared as mentioned above in order to confirm the sex of the para- 


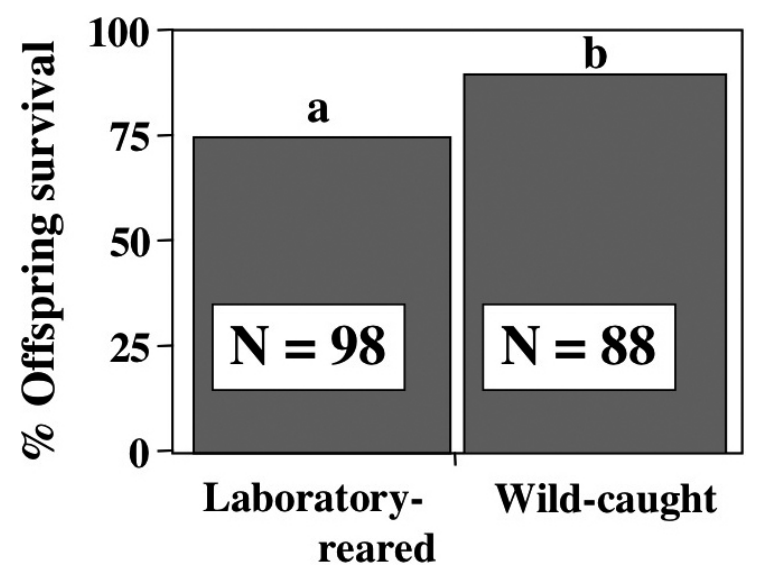

Fig. 1. Percentage of offspring of $A$. lanceolatus that survived. The percentage of parasitoid offspring emerging from hosts is used as the index. Survival of the offspring of laboratory-reared females was significantly lower (see text for statistical details).

sitoid offspring. Other aspects of the experimental set-up were the same as in the above experiment.

\section{Analyses}

Data were analyzed using JMP (SAS Institute, 2009). ANCOVAs were used to analyze the effect of host weight (covariate) and laboratory rearing (factor) on offspring survival and sex ratio. Simple and multiple logistic regression analyses were used to determine the relationships between offspring sex ratio and host weight.

\section{RESULTS}

\section{Host size}

In all, 212 hosts were presented to females of which 186 were parasitized. The survival of the offspring of both field and laboratory females of $A$. lanceolatus was high (>75\%) (Fig. 1). However, a multiple logistic regression analysis revealed that offspring parasitoid survival differed significantly between generations (Table 1). Host weight and interactions between host weight and parasitoid generation did not significantly affect offspring survival (Table 1; Fig. 2).

Because the effect of host weight on offspring parasitoid survival was not significant, data were pooled prior to calculating the percentage offspring survival for field and laboratory reared females. The survival of parasitoid offspring recorded for the laboratory-reared females was lower than that for the wild-caught females (Fig. 1) (chi-squared test; $x^{2}=8.98, P=0.0027$ ).

The results of the ANCOVA revealed that parasitoid offspring size was associated with host weight and parasitoid

TABLE 1. Multiple logistic regression analysis used to determine the effect of host weight and laboratory rearing on the survival of the offspring of $A$. lanceolatus.

\begin{tabular}{lccc}
\hline Factors & df & Wald x2 & P-value \\
\hline Host weight & 1 & 3.21 & 0.073 \\
Generation & 1 & 6.21 & 0.012 \\
Interaction & 1 & 0.09 & 0.77 \\
\hline Whole model was significant $\left(\mathrm{df}=3, r^{2}=0.08, x^{2}=12.92, P=\right.$ \\
0.0048).
\end{tabular}

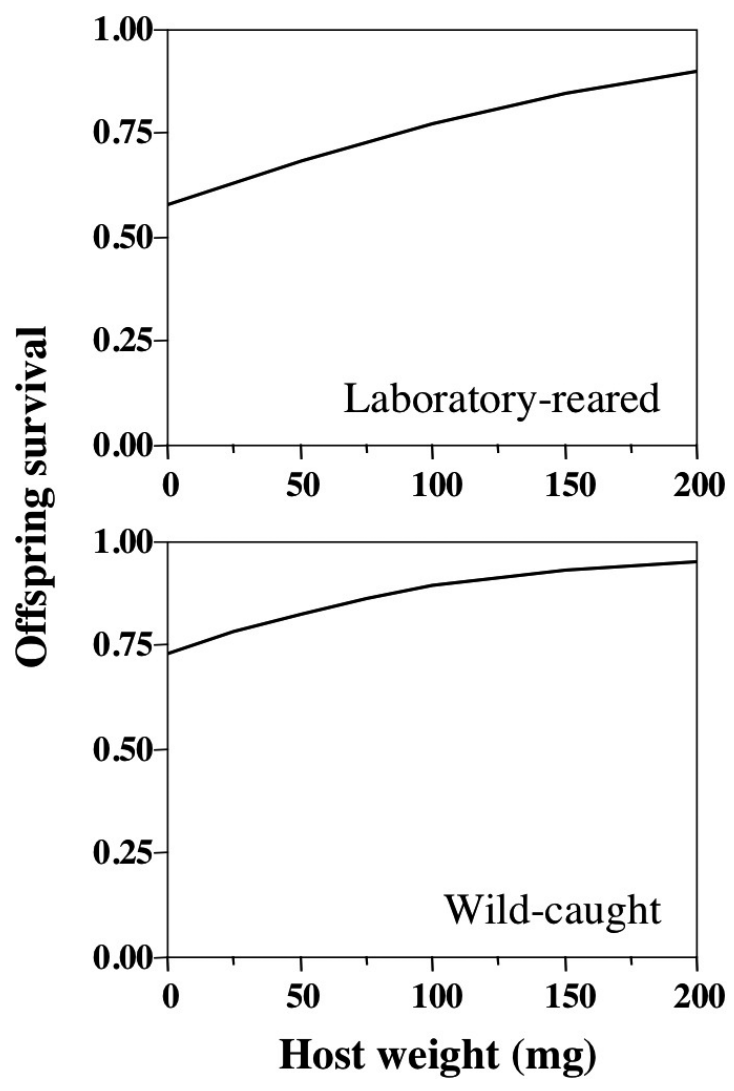

Fig. 2. Logistic regression analyses of the host-weight/hostsuitability relationships. Regressions were not significant for both parasitoid generations $(\mathrm{P}>0.10)$.

sex (Table 2). The interaction between these two factors was not significant. Parasitoid sex had a significant effect because the forewing length of females is longer than that of males. The size of emerging parasitoids increased with increasing host weight (Fig. 3) (Regression analysis; $\mathrm{N}=$ $31, r^{2}=0.48, \mathrm{~F}=27.0, P<0.0001$ for males; $\mathrm{N}=45, r^{2}=$ $0.69, \mathrm{~F}=97.6, P<0.0001$ for females).

Mean offspring sex ratio for the wild-caught females of A. lanceolatus was $74.1 \%$, whereas that for the laboratory-reared females was $41.9 \%$ (Fig. 4 ). The difference was highly significant (chi-squared test; $\mathrm{N}=155, x^{2}=16.8, P<$ $0.0001)$. Binomial tests revealed that the offspring sex ratio for the wild-caught females was significantly biased $\left(x^{2}=\right.$ 19.58, $P<0.0001)$ whereas that for the laboratory-reared females was not $\left(x^{2}=1.95, P=0.16\right)$. Thus, sex ratio of the offspring of wild-caught females was female-biased.

A multiple logistic regression analysis revealed that the sex of emerging A. lanceolatus was associated with host weight (Table 3 ). The $A$. lanceolatus sex ratio also differed

TABLE 2. Results of ANCOVA used to determine the effect of host weight on the size of the offspring (forewing length) of $A$. lanceolatus.

\begin{tabular}{lccc}
\hline Factors & df & F value & P-value \\
\hline Host weight & 1 & 97.41 & $<0.0001$ \\
Parasitoid sex & 1 & 4.47 & 0.038 \\
Interaction & 1 & 0.02 & 0.88 \\
\hline Whole model was highly significant & $\left(r^{2}=0.66, F=46.79, P<\right.$ \\
$0.0001)$. &
\end{tabular}



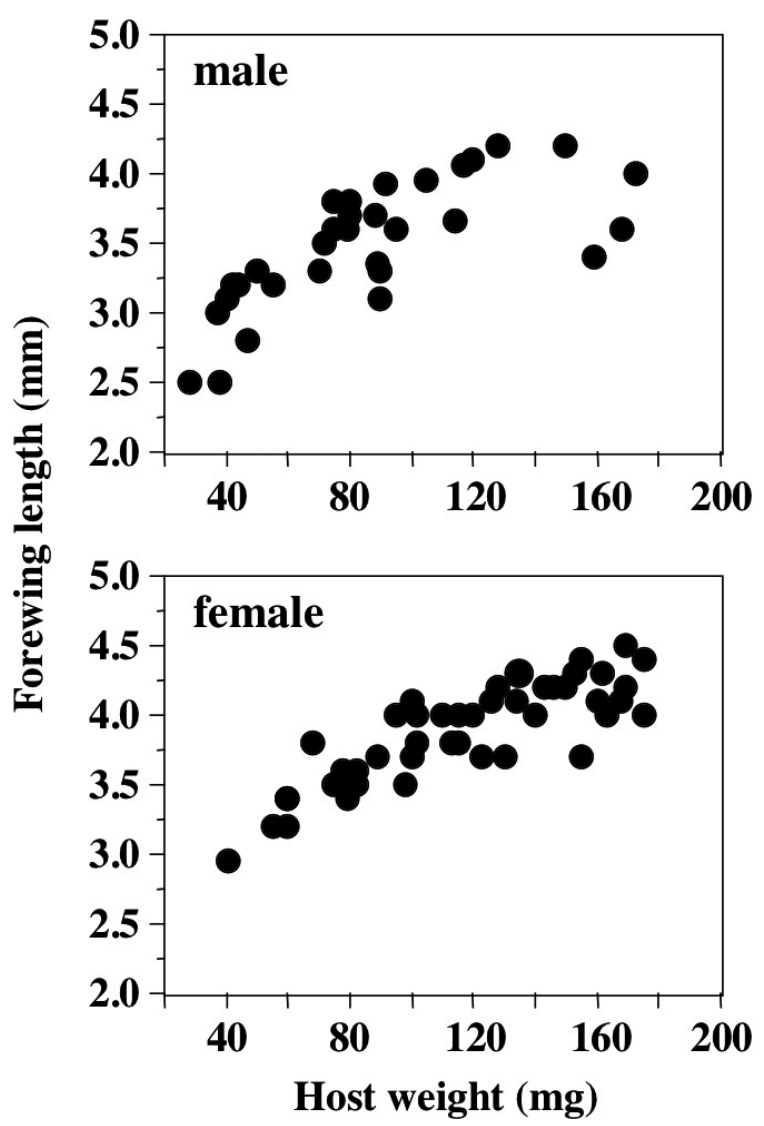

Fig. 3. Relationships recorded between emerging parasitoid size and host weight. For both males and females the relationships are positive (Regression analysis; $P<0.001$ ).

significantly for the field and laboratory reared females (Table 3). Simple logistic regression analyses revealed that the proportion of the emerging offspring of $A$. lanceolatus that were female increased with increase in host weight (Fig. 5) $\left(\mathrm{N}=81, r^{2}=0.24, x^{2}=22.1, P<0.0001\right.$ for the wild-caught females, $\mathrm{N}=74, r^{2}=0.22, x^{2}=22.3, P<$ 0.0001 for the laboratory-reared females). These analyses also revealed that the estimated host weight at which the parasitoid sex ratio was equal was $65.7 \mathrm{mg}$ for the wildcaught females and $102.6 \mathrm{mg}$ for the laboratory-reared females, respectively (Fig. 5)

\section{Primary sex-ratio}

In order to lay an egg female A. lanceolatus have to insert their ovipositor into a cocoon of a host. During ovipositor insertion, the base of the ovipositor moved rhythmically. The movements of the base of the ovipositor were of two types: continuous and interrupted. It was also possible to see an egg passing through the base of the ovipositor;

TABLE 3. Results of the multiple logistic regression analysis used to determine the effect of host weight and laboratory rearing on the sex ratio of the offspring of $A$. lanceolatus.

\begin{tabular}{lccc}
\hline Factors & df & Wald x2 & P-value \\
\hline Host weight & 1 & 28.83 & $<0.0001$ \\
Generation & 1 & 10.47 & 0.0012 \\
Interaction & 1 & 0.28 & 0.59 \\
\hline Whole model was highly significant $\left(r^{2}=0.29, x^{2}=61.20, P<\right.$ \\
0.0001).
\end{tabular}

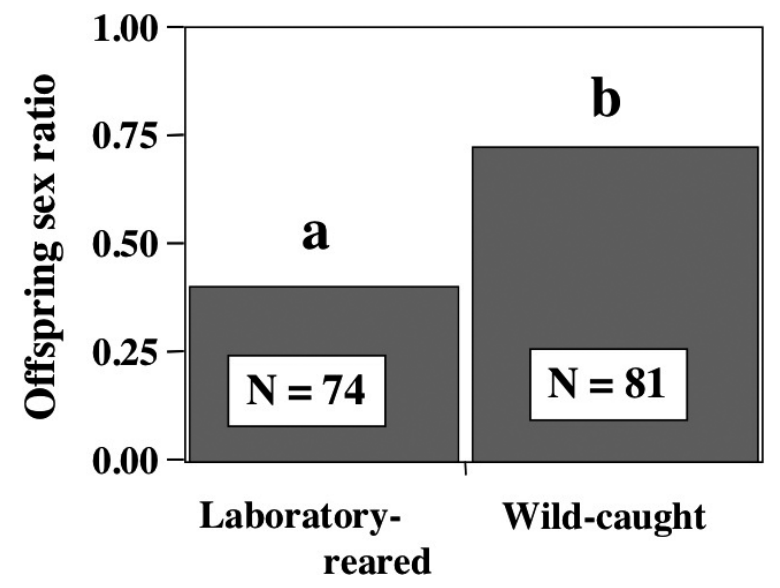

Fig. 4. Overall offspring sex ratio of $A$. lanceolatus. Offspring sex ratio differs significantly for field and laboratory reared females (Chi-squared test; $\mathrm{P}<0.05)$.

which confirmed that oviposition had occurred. Just prior to the egg being seen, it was frequently observed that the movements at the base of the ovipositor ceased at most for 2-3 s and then started again. In other cases the movements did not cease.

In total 40 of 42 hosts were attacked by the female parasitoids. Although in most cases the movements at the base of the ovipositor were either continuous or interrupted, in 2 cases, the movements were difficult to categorize because

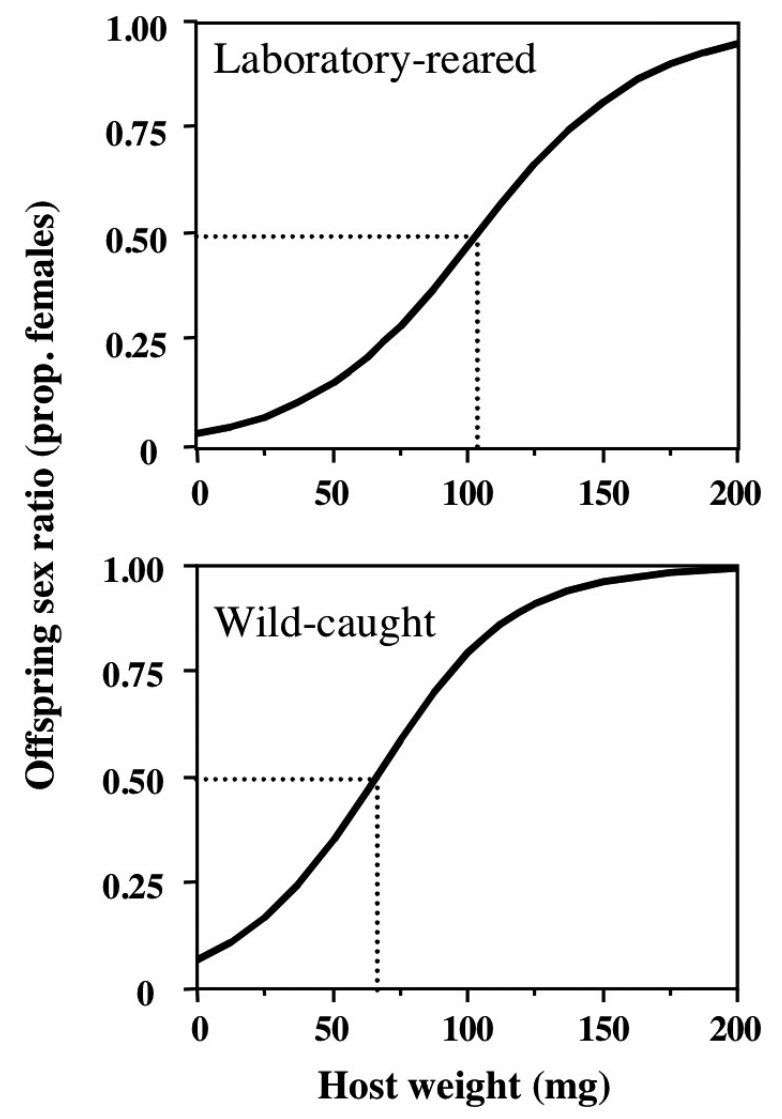

Fig. 5. Logistic regressions of the host weight/offspring sex ratio relationships. The regression lines are highly significant. There was a significant difference in the relationships for field and laboratory reared females (see Table 3 ). Dotted lines indicate host weights at which a $1: 1$ sex ratio is recorded. 


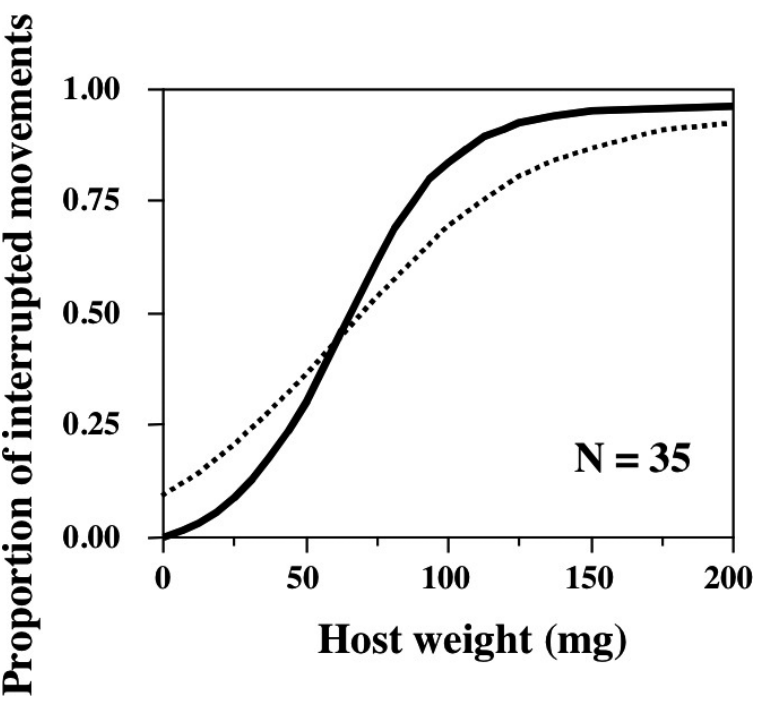

Fig. 6. Comparison of offspring sex ratio at the time of egg deposition (solid line) and that at adult parasitoid emergence (dashed line). Sex ratio at the egg stage is estimated on the basis of abdominal tip movements during oviposition (see text for details). The logistic regression line for sex ratio at the egg stage is highly significant.

they were too short. In 3 cases, females stood still with their ovipositor inserted in a host for more than $5 \mathrm{~min}$. In these cases, the observations were terminated before confirmation of oviposition and the data excluded from the analysis. Thus, in total 11 parasitoids were recorded as showing continuous and 24 interrupted movements and included in the following analyses.

The proportion showing interrupted movements increased with increasing host weight (Fig. 6; solid line) (logistic regression analysis; $\mathrm{N}=35, r^{2}=0.34, x^{2}=14.9$, $P=0.0001)$. Of the 35 hosts parasitized, 31 produced parasitoid offspring (offspring survival $=88.6 \%$ ). Hosts parasitized by parasitoids showing continuous movements produced only male parasitoids ( $100 \%$ accuracy), whereas the percentage of females was $90.5 \%$ for hosts parasitized by parasitoids showing interrupted movements. The percentage of female offspring based on the type of abdominal movement was $68.6 \%$ and did not differ from that of the offspring that emerged from the hosts (Chi-squared test; $x^{2}=0.38, P=0.54$ ). The parasitoid sex ratio (proportion of the female offspring that emerged $=$ secondary sex ratio) also increased with host weight (Fig. 6; dotted line) (logistic regression analysis; $\mathrm{N}=31, r^{2}=0.17, x^{2}=7.76$, $P=0.0053)$. The two logistic curves were similar. The pattern was also similar to that for the offspring sex ratio in response to host weight (Fig. 4). Male parasitoids emerged from hosts on which female parasitoids exhibited continuous movements during oviposition. In contrast, female parasitoid offspring emerged from hosts on which the mother parasitoids exhibited interrupted movements, although the control of fertilization was imperfect, which resulted in production of some males.

\section{DISCUSSION}

\section{Host suitability}

Small hosts are generally less suitable for parasitoid larval development, resulting in greater mortality and production of small parasitoid offspring, whose fitness (fecundity, longevity, etc.) is also usually low (King, 1987; Godfray, 1994; Quicke, 1997). Thus, the study of host-size/suitability relationships enables the identification of the most suitable host-size range for rearing parasitoids. In the current study, a wide range of hosts differing in weight were used to determine a host-size/suitability relationship for $A$. lanceolatus. The results indicate that $A$. lanceolatus can develop equally successfully in both small and large hosts (Table 1; Fig. 2) and that suitability of G. mellonella as a host does not depend on its size (Fig. 2). The results indicate that $A$. lanceolatus can parasitize a wide range of hosts of different sizes.

Host size had a strong effect on the size of the offspring of $A$. lanceolatus (Table 2) with large parasitoids emerging from large hosts (Fig. 3). Such a positive relationship has repeatedly been reported for the great majority of solitary parasitoids (King, 1987; Ueno, 1998, 1999a). The positive relationship between parasitoid size at emergence and host size is because large hosts contain more resources for the developing parasitoids. Thus, although G. mellonella cocoons of a range of different sizes are highly suitable for rearing $A$. lanceolatus, the use of small cocoons results in the production of small parasitoids. As small females of $A$. lanceolatus have short lives (Ueno, 1999b), it is preferable, wherever possible, to use large cocoons of $G$. mellonella as hosts.

\section{Evidence for the control of sex allocation}

Although many studies demonstrate a host-size-dependent sex-ratio at emergence in parasitoid wasps (King, 1987, 1993), relatively few provide direct evidence of maternal control of offspring sex-ratio. Experimental evidence so far consists of chromosome counts at the egg stage (Ueno \& Tanaka, 1997; Ueno, 1998b), detailed records of oviposition behaviour (Ueno, 1999a) and, more recently, the use of microsatellite DNA or RAPD markers to confirm the offspring sex at the egg stage (van Baaren et al., 1999; Abe et al., 2009). Signs indicating fertilization that can be inferred from abdominal tip movements during oviposition are reported for several hymenopteran parasitoids and sawflies, and are a useful tool for studying maternal sex-ratio control (Strand, 1989; Lee et al., 1998; Yamada \& Kawamura, 1999).

This paper presents evidence that the sex of deposited eggs can be determined by observing abdominal tip movements of ovipositing $A$. lanceolatus. The offspring sex ratios estimated by recording the movements at the base of the ovipositor of $A$. lanceolatus are host-size-dependent and consistent with the sex ratio of the emerging adults (Fig. 6). This indicates that the host-size-dependent sex ratio in A. lanceolatus is maternally controlled by controlling fertilization of the eggs. 
A curious result was obtained. The estimated offspring sex ratio appeared to be more female-based than that of the offspring at emergence. In fact, oviposition associated with continuous movements always resulted in the emergence of males whereas when associated with interrupted movements it did not always result in the emergence of females, but the accuracy was satisfactorily high (see the Results). Thus, the offspring sex ratio at the time of parasitoid emergence is more male-biased than that inferred from ovipositor tip movements.

Given that interrupted movements should indicate a maternal decision to fertilize and so lay a female egg, the results suggest that fertilization does not always occur, possibly due to physiological causes, which results in the laying of male eggs. The presence of physiological constraints on sex-ratio control has been suggested in a number of cases. A decrease in proportion of female offspring with maternal age is reported for a number of parasitoids, which has been attributed to shortage of sperm (Avilla \& Albajes, 1984; Ueno, 1995; Henter, 2004). Curiously, females of A. lanceolatus mate several times (T. Ueno, unpubl.) and, in my experiment, each female was allowed to mate only once. A single mating might not be enough for a female to lay female eggs throughout her adult life.

Alternatively, diploid males may be an explanation. Diploid males are recorded for a number of ichneumonids and other Hymenoptera (Quicke, 1997). If this is the case for $A$. lanceolatus, inbreeding in a laboratory culture could result in the production of diploid males, decreasing the proportion of female offspring while the estimated sex-ratio at oviposition remains constant. Unfortunately, in my sexratio experiment, care was not taken to avoid brother-sister mating. Factors causing a male-biased sex ratio at parasitoid emergence remain unresolved.

\section{Host-size-dependent sex ratio}

For many parasitoids, the proportion of males among emerging parasitoids declines as host size increases (King, 1987, 1993). Ovipositing females of A. lanceolatus are able to assess the size of host prepupae enclosed in cocoons and change offspring sex ratio accordingly. A striking result of the present study is the marked difference in offspring sex ratio in response to host size detected for the field and laboratory reared parasitoid females. A higher proportion of female offspring emerged from most classes of host size when wild-caught females rather than laboratory-reared females were used (Fig. 5). As a result, the mean offspring sex ratio of $A$. lanceolatus is female-biased for wild-caught females (Fig. 4). This difference is attributable either to differences in sex allocation behaviour in field and laboratory reared females or inbreeding depression during laboratory rearing, or both.

Female age influence sex allocation in a number of parasitoids (Avilla \& Albajes, 1984; King, 1987; Simbolotti et al., 1987). If this is the case for A. lanceolatus, the difference in the ages of the field and laboratory females might account for the differences in offspring sex ratio. Age of wild caught females used in the present study was not known, although the unworn condition of their wings indi- cated they were young. Old females are known to produce a more male-biased offspring sex ratio (Avilla \& Albajes, 1984). In fact, a female-biased sex ratio was recorded for wild-caught females (Fig. 4). This indicates that the difference in female age is not the explanation.

Alternatively, inbreeding depression may be the explanation. In this case, two processes could be involved: increased mortality of female offspring and production of diploid males. Offspring mortality is considerably higher when laboratory-reared rather than wild-caught females are the mothers (Figs 1 and 2). This may be because female offspring are more likely to die than the male offspring of laboratory-reared females. An increase in the production of diploid males may also be the reason. Diploid males are reported in a number of ichneumonid wasps when inbreeding occurs in species with CSD systems (Quicke, 1997; Heimpel \& Lundgren, 2000; Ode \& Heinz, 2002). Male-biased sex ratios are reported for many commercially reared parasitoids, and inbreeding is the usual explanation (Ueno, 1999c; Heimpel \& Lundgren, 2000). In many cases, factors leading to differences in sex ratios of field and laboratory females remain to be resolved.

It is well known that a number of biological parameters change during the mass rearing of insect natural enemies, including parasitoids. Offspring survival and sex ratio are thought to be among such parameters (Jervis \& Kidd, 1996; Heimpel \& Lundgren, 2000; Ode \& Heinz, 2002). Nevertheless, few studies have examined the process by which such changes occur during mass rearing. Very few studies have focused on changes in biological parameters from the initial establishment of a culture, i.e., parameters for F1 generations (Ueno, 1999c). However, determining the biological parameters of F1 generations is important because knowing the qualitative and quantitative differences between mass-reared natural enemies and wild natural enemies is crucial for assessing the quality of massreared natural enemies.

ACKNOWLEDGEMENTS. The author thanks two anonymous reviewers and A.F.G. Dixon for their valuable suggestions to improve the manuscript.

\section{REFERENCES}

Abe J., Kamimura Y., Shimada M. \& West S.A. 2009: Extremely female-biased primary sex ratio and precisely constant male production in a parasitoid wasp Melittobia. - Anim. Behav. 78: $515-523$.

Avilla J. \& Albajes R. 1984: The influence of female age and host size on the sex ratio of the parasitoid Opius concolor. Entomol. Exp. Appl. 35: 43-47.

Charnov E.L. \& Sikinner S.W. 1984: Evolution of host selection and clutch size in parasitoid wasps. - Fla Entomol. 67: 5-21.

Charnov E.L., Harthigh R.L., Jones W.T. \& Van Alphen J. 1981: Sex ratio evolution in a variable environment. - Nature 289: 27-33.

Godfray H.C.J. 1994: Parasitoids: Behavioral and Evolutionary Ecology. Princeton University Press, Princeton, 488 pp.

Goubault M.E., Fourrier J., Krespi D., Poinsot D. \& Cortesero A.M. 2004: Selection strategies of parasitized hosts in a generalist parasitoid depend on patch quality but also on host size. -J. Insect Behav. 17: 99-113. 
HajeK A. 2004: Natural Enemies: An Introduction to Biological Control. Cambridge University Press, Cambridge, 396 pp.

Heimple G.E. \& Lundgren J.G. 2000: Sex ratios of commercially reared biological control agents. - Biol. Contr. 19: 77-93.

Henter H.J. 2004: Constrained sex allocation in a parasitoid due to variation in male quality. - J. Evol. Biol. 17: 886-896.

Jervis M.A. \& KidD N.A.C. 1996: Insect Natural Enemies. Chapman \& Hall, London, 491 pp.

JMP 2009: JMP Version 9.0. SAS Institute Inc., Cary, NC.

KING B.H. 1987: Offspring sex ratios in parasitoid wasps. Quart. Rev. Biol. 62: 367-396.

KING B.H. 1993: Sex ratio manipulation by parasitoid wasps. In Wrensch D.L. \& Ebbert M. (eds): Evolution and Diversity of Sex Ratio in Insects and Mites. Chapman \& Hall, New York, pp. $418-441$

Lee J.M., Hashino Y., Hatakeyama M., Oishi K. \& Naito T. 1998 Egg deposition behavior in the haplodiploid sawfly Atalia rosae ruficornis Jakovlev (Hymenoptera: Symphyta: Tenthredinidae). - J. Insect Behav. 11: 419-428.

Liu Z., Xu B. \& Sun J. 2011: Host-size mediated trade-off in a parasitoid Sclerodermus harmandi. — PlosONE 6: e23260.

Mao H. \& Kunimi Y. 1991: Pupal mortality of the oriental tea tortrix, Homona magnanima Diakonoff (Lepidoptera: Tortricidae), caused by parasitoids and pathogens. - Jap. J. Appl. Entomol. Zool. 35: 241-245.

Ode P.J. \& Heinze K.M. 2002: Host-size-dependent sex ratio theory and improving mass-reared parasitoid sex ratios. - Biol. Cont. 24: 31-41.

Quicke D.L.J. 1997: Parasitic Wasps. Chapman \& Hall, London, $470 \mathrm{pp}$.

Rehman A. \& Powell W. 2010: Host selection behavior of aphid parasitoids (Aphidiidae: Hymenoptera). — J. Plant Breed Crop Sci. 2: 299-311.

RYAN R.B. 1971: Interaction between two parasites, Aphechthis ontario and Itoplectis quadricingulatus. - Ann. Entomol. Soc. Am. 64: 205-208.

SANDLAN K. 1982: Host suitability and its effects on biology of Coccygomimus turionellae (Hymenoptera: Ichneumonidae). - Ann. Entomol. Soc. Am. 75: 217-221.

Simbolotti G., Putters F.A. \& Van Alphen J. 1987: Rates of attack and control of the offspring sex ratio in the parasitic wasp Lariophagus distinguendus in an environment where host quality varies. - Behaviour 100: 1-32.

STRAND M.R. 1989: Oviposition behavior and progeny allocation of the polyembryonic wasp Copidosoma floridanum (Hymenoptera: Encyrtidae). - J. Insect Behav. 2: 355-368.

Townes H., Momoi S. \& Townes M. 1965: A Catalogue and Reclassification of the Eastern Palearctic Ichneumonidae. Memoirs of the American Entomological Institute 5, $671 \mathrm{pp}$.

TRAYNOR R.E. \& MAYHEW P.J. 2005: Host range in solitary versus gregarious parasitoids: a laboratory experiment. — Entomol. Exp. Appl. 117: 41-49.
UENo T. 1995: Abdominal tip movements during oviposition by two parasitoids (Hymenoptera: Ichneumonidae) as an index of predicting the sex of depositing eggs. - Appl. Entomol. Zool. 30: $588-590$.

Ueno T. 1998a: Adaptiveness of sex ratio control by the pupal parasitoid Itoplectis naranyae (Hymenoptera: Ichneumonidae) in response to host size. - Evol. Ecol. 12: 643-655.

Ueno T. 1998b: Sex allocation responses by a parasitoid wasp (Hymenoptera: Ichneumonidae) to different host species: a question for the mechanism of host size estimation. - J. Insect Behav. 11: 811-821.

Ueno T. 1999a: Host-size-dependent sex ratio in a parasitoid wasp. - Res. Popul. Ecol. 41: 47-57.

UENO T. 1999b: Adult size and reproduction in the ectoparasitoid Agrothereutes lanceolatus Walker (Hym., Ichneumonidae). J. Appl. Entomol. 123: 357-362.

UENO T. 1999c: Host suitability and sex ratio differences in wildcaught and laboratory reared parasitoid Pimpla parnarae (Hymenoptera: Ichneumonidae). - Ann. Entomol. Soc. Am. 92: 609-614.

UENO T. 2000: Host concealment: a determinant for host acceptance and feeding in an ectoparasitoid wasp. - Oikos 89: 223-230.

UENo T. 2002: Biology of the ectoparasitoid wasp Agrothereutes lanceolatus Walker (Hym., Ichneumonidae): Host acceptance and larval development on a laboratory host. - J. Fac. Agr. Kyushu Univ. 47: 37-43.

Ueno T. \& TANAKA T. 1997: Comparison of primary and secondary sex ratios in parasitoid wasps using a method for observing chromosomes. - Entomol. Exp. Appl. 82: 105-108.

VAn BaARen J., Landry B.L. \& Boivin G. 1999: Sex allocation and larval competition in a superparasitizing solitary egg parasitoid: competing strategies for an optimal sex ratio. - Funct. Ecol. 13: 66-71.

Vinson S.B. \& IwANTSCh G.F. 1980a: Host regulation by insect parasitoids. - Quart. Rev. Biol. 55: 143-165.

VINSON S.B. \& IwANTSCH G.F. 1980b: Host suitability for insect parasitoids. - Annu. Rev. Entomol. 25: 397-419.

Wang X.G \& Messing R.H. 2004: Fitness consequences of bodysize-dependent host species selection in a generalist ectoparasitoid. — Behav. Ecol. Sociobiol. 56: 513-322.

YAMADA Y.Y. \& KaWAMURA M. 1999: Sex identification of eggs of a dryinid parasitoid, Haplogonatopus atratus, based on oviposition behaviour. - Entomol. Exp. Appl. 93: 321-324.

Yasumatsu K. \& Watanabe C. 1965: A Tentative Catalogue of Insect Natural Enemies of Injurious Insects in Japan. Part 2. Host Parasite-predator Catalogue. Entomol. Lab. Fac. Agric. Kyushu Univ., Fukuoka, 116 pp.

Received July 14, 2014; revised and accepted October 29, 2014 Prepublished online February 27, 2015 
